\title{
TU/e EmonOWEN

\section{Experimental validation of the stochastic model of a randomly fluctuating transmission-line}

\section{Citation for published version (APA):}

Sy, O. O., Vaessen, J. A. H. M., Beurden, van, M. C., Michielsen, B. L., Tijhuis, A. G., Zwamborn, A. P. M., \& Groot, J. (2008). Experimental validation of the stochastic model of a randomly fluctuating transmission-line. In Microwave Conference, 2008. EuMC 2008. 38th European, 27-31 Oct. 2008, London, UK (pp. 833-836). Institute of Electrical and Electronics Engineers. https://doi.org/10.1109/EUMC.2008.4751582

DOI:

10.1109/EUMC.2008.4751582

Document status and date:

Published: 01/01/2008

\section{Document Version:}

Publisher's PDF, also known as Version of Record (includes final page, issue and volume numbers)

\section{Please check the document version of this publication:}

- A submitted manuscript is the version of the article upon submission and before peer-review. There can be important differences between the submitted version and the official published version of record. People interested in the research are advised to contact the author for the final version of the publication, or visit the $\mathrm{DOI}$ to the publisher's website.

- The final author version and the galley proof are versions of the publication after peer review.

- The final published version features the final layout of the paper including the volume, issue and page numbers.

Link to publication

\section{General rights}

Copyright and moral rights for the publications made accessible in the public portal are retained by the authors and/or other copyright owners and it is a condition of accessing publications that users recognise and abide by the legal requirements associated with these rights.

- Users may download and print one copy of any publication from the public portal for the purpose of private study or research.

- You may not further distribute the material or use it for any profit-making activity or commercial gain

- You may freely distribute the URL identifying the publication in the public portal.

If the publication is distributed under the terms of Article 25fa of the Dutch Copyright Act, indicated by the "Taverne" license above, please follow below link for the End User Agreement:

www.tue.nl/taverne

Take down policy

If you believe that this document breaches copyright please contact us at:

openaccess@tue.nl

providing details and we will investigate your claim. 


\title{
Experimental validation of the stochastic model of a randomly fluctuating transmission-line
}

\author{
O.O. Sy ${ }^{\# 1}$, J.A.H.M. Vaessen \#, M.C. van Beurden *, B.L. Michielsen *, \\ A.G. Tijhuis ${ }^{*}$, A.P.M. Zwamborn ${ }^{\dagger}$, J. Groot ${ }^{\dagger}$ \\ \# Electromagnetics Department, Eindhoven, University of Technology \\ Den Dolech 5, 5612 AZ, The Netherlands \\ 1 o.o.sy@tue.nl \\ * ONERA DEMR \\ Electromagnetics and Radar Department \\ 2, avenue Edouard Belin, 31055 TOULOUSE cedex, France \\ ${ }^{\dagger}$ TNO Defence, Security and Safety \\ Oude Waalsdorperweg 632597 AK Den Haag, The Netherlands
}

\begin{abstract}
A modeling method is proposed to quantify uncertainties affecting electromagnetic interactions. This method considers the uncertainties as random and measures them thanks to probability theory. A practical application is considered through the case of a transmission-line of varying geometry, illuminated by a fixed electromagnetic field. The results of the stochastic numerical model are compared to the measurements performed on the transmission-line setup.
\end{abstract}

\section{INTRODUCTION}

Real-life electromagnetic interactions are often affected by uncertainties. These uncertainties may be due to uncontrolled changes caused by manufacturing tolerances, mechanical vibrations, ageing or fatigue. In some other cases, uncertainties are caused by the complexity of the setup which leads to a lack of knowledge of the actual value of the parameters describing the configuration of the interaction. This is typically the case when studying the internal electromagnetic compatibility of complex electronic devices such as integrated circuits. More generally, such problems arise in coupling phenomena between objects of varying or unknown shape, together with incident fields created by electromagnetic sources in their environment.

To model such situations, these uncertainties need to be accounted for. Doing so by studying every possible configuration is extremely costly if not impossible. Another approach consists in considering the variations of the input parameters as random. The output parameters resulting from the electromagnetic interaction, also known as "observables", then become random variables. The theory of probability then allows to measure the uncertainty of the observables, either by their probability distribution, which is the ideal measure, but difficult to determine explicitly, or by the statistical moments of the observables, which are computable. Although these moments do not provide a complete statistical characterization, they yield valuable qualitative and quantitative information on the distribution of the observable. Moreover, the average and the variance fully characterize the probability distribution of random variables of practical importance, viz. Gaussian random variables.

Such an approach has already been tried in mode-stirredchamber theory where statistical methods are used to describe the random illumination incident on deterministic objects as is done by Hill [1]. Bellan and Pignari [2] applied a probabilistic method to study a deterministic wire structure under random plane-wave illumination by using transmission-line theory. In [3], we have proposed a stochastic approach to tackle uncertainties in electromagnetic problems modeled by integral equations. A 1-meter long randomly undulating transmissionline over a ground plane was studied as an application. We computed the mean and the variance of the voltage induced at the port of this transmission-line by a deterministic plane wave. This setup, derived from an EMC benchmark, is representative for a wire connection to an integrated circuit, or for a transmission-line lying on the ground such as those commonly used in telecommunications. Moreover, owing to the scalability of Maxwell's equations, the model derived for this macroscopic configuration can be adapted to setups of smaller dimensions.

The aim of the present paper is to verify the validity of the assumption of the latter stochastic model, by comparing it to the experimental results of a practical setup.

The outline of this paper is as follows. The model used in the simulations is introduced in Section II. A deterministic description of the setup is given first, where the shape of the scatterer and the incident field are presented. This configuration is then randomly parameterized by assuming the geometrical variations to be random. The average and variance of the induced voltage derived from this numerical model are computed by using two different quadrature rules: a Monte-Carlo rule, and a Cartesian-product rule. Section III details the practical configuration that is used as a test case, as well as the protocol followed during the measurements. These experimental results are compared to the simulated ones, and discussed in Section IV. 


\section{Simulation MODEL}

A deterministic model of the setup is first established to numerically represent the electromagnetic interaction. The random parametrization of this model then allows to use probability theory to measure the dispersion in the values of the induced voltage.

\section{A. Deterministic model}

The thin wire is represented by a perfectly electrically conducting (PEC) wire, with a radius $a=1 \mathrm{~mm}$. The wire lies on top of a PEC ground plane which is assumed to be of infinite extent as depicted in Figure 1.

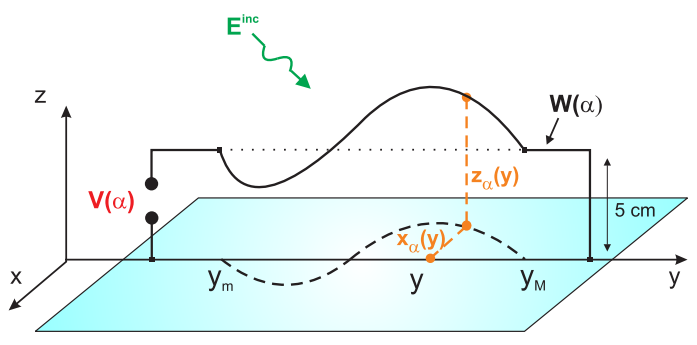

Fig. 1. Geometrical model of the setup

The wire consists of two vertical posts of $5 \mathrm{~cm}$ length, which are connected to the ground plane below and to a "horizontal" thin wire above. One of the posts contains a port region denoted $P$, and represented by a $2 \mathrm{~cm}$-large gap located 1.5 $\mathrm{cm}$ above the ground plane. The transverse position of the "horizontal" wire is assumed to be fluctuating over a distance $L_{y}=y_{M}-y_{m}=1 m$ between the abscissae $y=y_{m}$ and $y=y_{M}$. The points of the axis of this wire are represented by their Cartesian coordinates $(x(y), y, z(y))$. For the sake of generality, a Fourier representation of the wire is adopted meaning that $x(y)$ and $z(y)$ are written as Fourier sums

$$
\begin{aligned}
& x(y)=\sum_{k=1}^{N_{x}} \alpha_{x, k} \sin \left(k \pi \frac{y-y_{m}}{L_{y}}\right)=x_{\alpha}(y) \\
& z(y)=\sum_{k=1}^{N_{z}} \alpha_{z, k} \sin \left(k \pi \frac{y-y_{m}}{L_{y}}\right)=z_{\alpha}(y)
\end{aligned}
$$

where the coefficients $\alpha_{x, k} \in \Omega_{x, k}$ and $\alpha_{z, k} \in \Omega_{z, k}$ for each $k$. The coefficients $\alpha_{x, k}$ (resp. $\alpha_{z, k}$ ) vary in their domains $\Omega_{x, k}$ (resp. $\Omega_{z, k}$ ). The bounds of the domains $\Omega_{x, k}$ and $\Omega_{z, k}$ are dictated by the setup. For instance, the sets $\Omega_{z, k}$ are such that the wire never goes through the ground plane, i.e. $z_{\alpha}(y) \geq 0$. The vector $\boldsymbol{\alpha}$ gathers all the coefficients $\alpha_{x, k}$ and $\alpha_{z, k}$, and therefore belongs to the domain $\Omega_{\boldsymbol{\alpha}}$ given by the Cartesian product of all the domains $\Omega_{x, k}$ and $\Omega_{z, k}$. The entire wire is coined $W(\boldsymbol{\alpha})$ to indicate that its shape varies with $\boldsymbol{\alpha}$.

The incident field $\boldsymbol{E}^{i}$ is represented by a plane wave. It represents the field radiated by the source used in the experimental setup, in absence of the wire frame. This model of an incident field is suitable as long as the wire frame lies in a region far from the sources.
The voltage $V_{e}$ induced by $\boldsymbol{E}^{i}$ at the port $P$ is obtained by the superposition of the voltage $V_{i}$, due to the direct interaction between $P$ and $\boldsymbol{E}^{i}$, and the voltage $V_{s}(\boldsymbol{\alpha})$ which arises from the coupling between $P$ and the field $\boldsymbol{E}^{s}$ scattered by $W(\boldsymbol{\alpha})$.

$$
V_{e}(\boldsymbol{\alpha})=V_{i}+V_{s}(\boldsymbol{\alpha})
$$

As detailed in [1], $V_{s}(\boldsymbol{\alpha})$ can be expressed as a reaction integral between $\boldsymbol{E}^{i}$ and a transmitting-state current $\boldsymbol{j}_{\boldsymbol{\alpha}}$. The current $j_{\boldsymbol{\alpha}}$, which is created when a unitary current source is placed at the port $\mathrm{P}$ in absence of $\boldsymbol{E}^{i}$, is obtained by solving an electric-field integral equation via the method of moments.

\section{B. Random parametrization}

Since $V_{s}(\boldsymbol{\alpha})$ depends on $\boldsymbol{\alpha}$, the variations of the parameters $\boldsymbol{\alpha}$ in $\Omega_{\boldsymbol{\alpha}}$ translate into fluctuations of $V_{e}(\boldsymbol{\alpha})$ in a domain $\Omega_{V_{e}}$. To measure these fluctuations by using probability theory, a random parametrization is performed.

To begin with, $\boldsymbol{\alpha}$ is assumed to vary randomly in $\Omega_{\boldsymbol{\alpha}}$ according to a known probability distribution $p_{\boldsymbol{\alpha}}$. This distribution, chosen a priori, depicts the spread of the values of $\boldsymbol{\alpha}$ in $\Omega_{\boldsymbol{\alpha}}$. If all values of $\Omega_{\boldsymbol{\alpha}}$ are equally likely to occur, a uniform distribution is chosen. On the other hand, if the average and the variance of $\boldsymbol{\alpha}$ are prescribed, a normal distribution may be used for $\boldsymbol{\alpha}$.

As a result, $V_{e}(\boldsymbol{\alpha})$ becomes a random variable which takes its values in $\Omega_{V_{e}}$. Since the probability distribution $p_{V_{e}}$ of $V_{e}(\boldsymbol{\alpha})$ can usually not be computed as a function of $p_{\boldsymbol{\alpha}}$, the objective is instead to determine the statistical moments of $V_{e}(\boldsymbol{\alpha})$. The average of $V_{e}(\boldsymbol{\alpha})$, for instance, is defined as

$$
\mathbb{E}\left[V_{e}\right]=\int_{\Omega \boldsymbol{\alpha}} V_{e}\left(\boldsymbol{\alpha}^{\prime}\right) p_{\boldsymbol{\alpha}}\left(\boldsymbol{\alpha}^{\prime}\right) d \boldsymbol{\alpha}^{\prime} .
$$

The right-hand side of this equation is computable: it involves an integral over a known domain $\Omega_{\boldsymbol{\alpha}}$, and a known integrand $V_{e}\left(\boldsymbol{\alpha}^{\prime}\right) p_{\boldsymbol{\alpha}}\left(\boldsymbol{\alpha}^{\prime}\right)$. The variance of $V_{e}$ is defined likewise as

$$
\sigma\left[V_{e}\right]^{2}=\mathbb{E}\left[\left|V_{e}\right|^{2}\right]-\left|\mathbb{E}\left[V_{e}\right]\right|^{2} .
$$

Due to the intricate expression of $V_{e}(\boldsymbol{\alpha})$ in terms of $\boldsymbol{\alpha}$, the integrals in Equations (4) and (5) usually cannot be determined in closed form. They are instead computed numerically via a Cartesian-product quadrature rule. This rule requires $N_{\text {cart }}$ deterministic samples in $\Omega_{\boldsymbol{\alpha}}$. The convergence rate of this rule decreases as $1 / N_{\text {cart }}^{2}$ for low-order repeated rules. The value of $N_{\text {cart }}$ however depends exponentially on the dimension of $\Omega_{\boldsymbol{\alpha}}$. Further details on these rules can be found in [3] and [4].

In the results provided below, several choices are illustrated for the model of the geometry by considering different values for $N_{x}$ and $N_{z}$ in Equations (1) and (2). The average and variance that are computed are then compared to the statistical moments obtained experimentally. The gaussian distribution corresponding to the simulated moments is compared to the empirical distribution obtained from the experimental data, in order to check whether the voltage $V_{e}$ can be considered as a gaussian random variable. 


\section{DESCRIPTION OF THE EXPERIMENTAL SETUP}

After having defined the wire model that is suitable for computing the influence of a varying geometry on the induced voltage, it is interesting to compare the results that have been obtained through simulations by means of measurements on a real structure. At TNO Defence, Security and Safety in The Hague, a measurement setup has been constructed for the wire structure. A photograph of the configuration is shown in Fig. 2. The ground plane is made of brass and its dimensions are $[1 \mathrm{xb}=1 \mathrm{~m} \times 2 \mathrm{~m}]$. The wire is made from a woven mantle stripped from a coaxial cable and has a diameter of $1 \mathrm{~mm}$. The total length of the wire is approximately 1.12 meters. This includes the part of the wire located in the vertical posts. If the wire is pulled tight, the distance between wire and ground plane is 0.05 meter. The length of the wire can be increased or decreased to create a flexible, or stiff wire which influences the freedom of movement. The wire structure is excited by a so-called biconolog, antenna which is located at approximately 3.2 meters from the wire structure. The height above the ground level and polarization of the antenna can be adjusted at will.

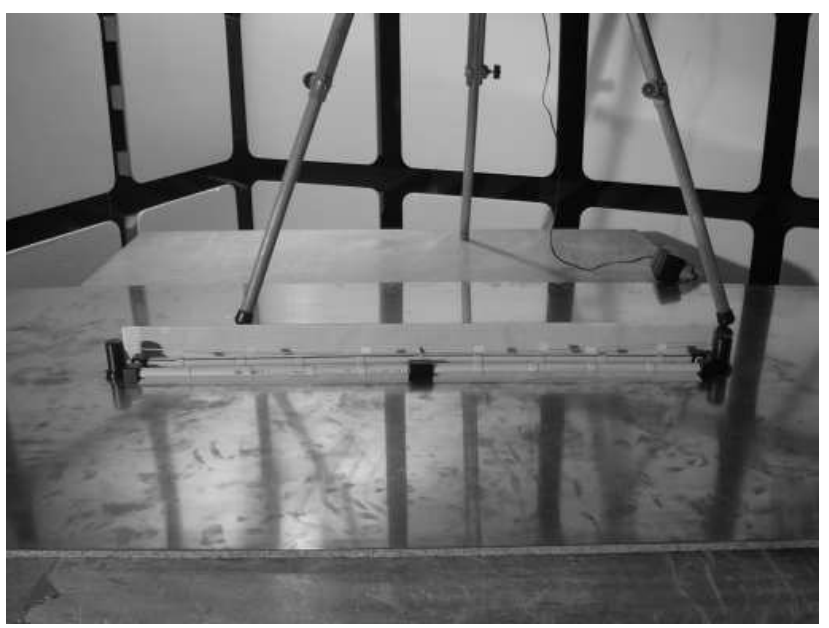

Fig. 2. Setup of the experiment

A challenging problem is to come up with a setup in which the geometry of the wire is able to vary stochastically. To this end two PVC tubes, in which small holes are drilled, are mounted on the ground plane at both sides below the wire. Through these PVC tubes high pressure air is blown towards the wire. Further, small paper flags have been attached to the wire to i) increase the force applied by the air to the wire and ii) stimulate stochastic movement. Through the control of the air flow, an adjustable stochastic variation of the wire is obtained. The rms value of the induced voltage is measured by means of a LeCroy oscilloscope. Further, the movement of the wire is recorded by two high-speed cameras, one located in front of the wire structure and one above the wire structure. In this way it is possible, to reconstruct the three dimensional time varying shape of the wire and to compare it to computations. Several measurements have been performed in steady state with and without cameras to investigate the influence of the cameras on the measurements. We have observed little difference in the measured voltage with or without the presence of the cameras.

\section{RESULTS}

\section{A. Measurement results}

The structure is excited by the biconolog antenna at a frequency of $500 \mathrm{MHz}$. The incident field is horizontally polarized. The steady state of the wire configuration, i.e., without air flow, is shown in Fig. 3 We have chosen for this

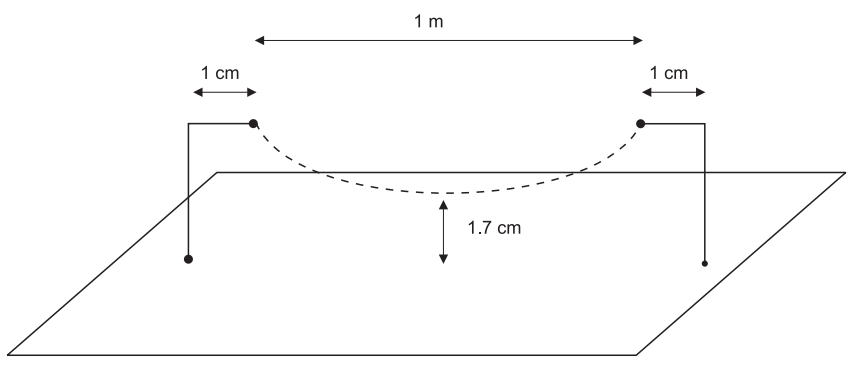

Fig. 3. Steady state configuration

particular situation to allow the wire to be very flexible and therefore have a large freedom of movement. By enabling the air flow, the geometry of the wire changes rapidly, simulating a stochastic geometry. First, for calibration purposes, the induced voltage in steady state is measured. Secondly, the air flow is enabled and the effect of the changing geometry on the induced voltage is measured. The induced voltage is measured during 5 seconds by the LeCroy oscilloscope taking 10.000 samples per second. With this configuration the number of possible geometries approximates a random set. Fig. 4

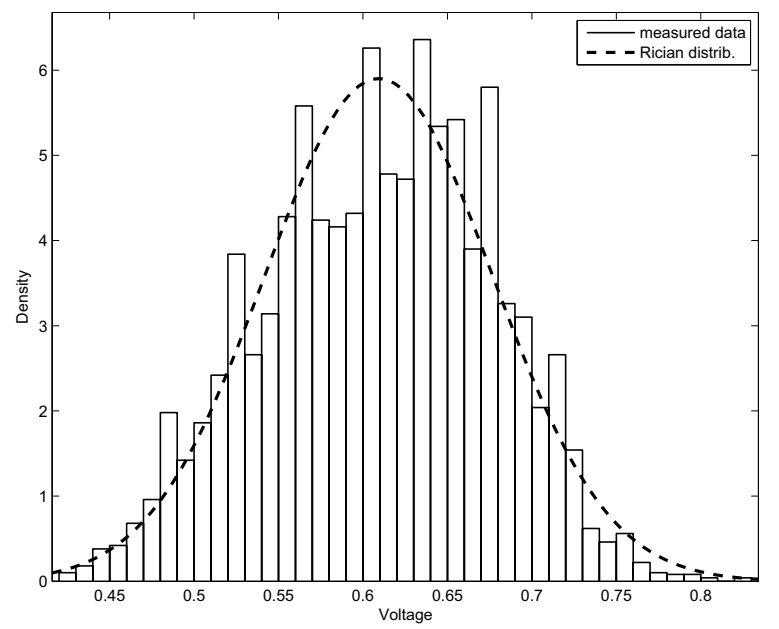

Fig. 4. Density of the measured voltages together with a Rician distribution with $\mu_{e x p}=0.6079 \mathrm{~V}$ and $\sigma_{e x p}=0.0676 \mathrm{~V}$, which have been obtained from the measurement data.

shows the distribution of the measured voltage as well as a 
Rician distribution with the measured mean and variance as input parameters. This plot shows good agreement between the Rician distribution and the measurement data. From the theory of statistics [5], we know that if two independent random variables have a Gaussian distribution (e.g., the real and imaginary part of the spectrum of the voltage), their module should have a Rician distribution.

\section{B. Simulation Results}

A horizontally-polarized plane-wave is considered as incident field. The parameters of this excitation are chosen in agreement with the characteristics of the source described in section III.

As can be seen in Table I, several types of geometries are taken into account for the transmission-line $W(\boldsymbol{\alpha})$, by choosing different values for the parameters $N_{x}$ and $N_{z}$ in Equations (1) and (2). The length and the stiffness of the thin-wire impose that $x_{\boldsymbol{\alpha}}(y) \in[-3.3 ; 3.3] \mathrm{cm}$ and $z_{\boldsymbol{\alpha}}(y) \in$ $[5-3.3 ; 5+3.3] \mathrm{cm}$. The vector $\boldsymbol{\alpha}$ is assumed to be have mutually independent and uniformly distributed components.

TABLE I

DIFFERENT TYPES OF GEOMETRICAL MODELS

\begin{tabular}{|c|c|c|}
\hline Wire $W_{N_{x}, N_{z}}(\boldsymbol{\alpha})$ & $\Omega_{x, k}$ & $\Omega_{z, k}$ \\
\hline$W_{1,1}(\boldsymbol{\alpha})$ & {$[-3.3 ; 3.3] \mathrm{cm}$} & {$[1.7 ; 8.3] \mathrm{cm}$} \\
$W_{1,2}(\boldsymbol{\alpha})$ & {$[-3.3 ; 3.3] \mathrm{cm}$} & {$[3.35 ; 6.65] \mathrm{cm}$} \\
\hline
\end{tabular}

For each of these models, the average and the standard deviation of the module of the voltage is computed. These results are gathered in Table II where they are compared to the experimental results $\mu_{\text {exp }}=607.9 \mathrm{mV}$ and $\sigma_{\text {exp }}=67.6$ $\mathrm{mV}$, via the relative errors $\varepsilon_{1}$ for the average, and, $\varepsilon_{2}$ for the standard deviation. These results first highlight the appreciable

TABLE II

Simulated STATISTICS OF THE VOLTAGE

\begin{tabular}{|l||c|c||c|c|}
\hline & $\mathbb{E}[|V|]$ & $\varepsilon_{1}$ & $\sigma[V]$ & $\varepsilon_{2}$ \\
\hline$W_{1,1}(\boldsymbol{\alpha})$ & $540.1 \mathrm{mV}$ & $11.1 \%$ & $68.3 \mathrm{mV}$ & $1 \%$ \\
$W_{1,2}(\boldsymbol{\alpha})$ & $537.5 \mathrm{mV}$ & $11.6 \%$ & $67.3 \mathrm{mV}$ & $0.4 \%$ \\
\hline
\end{tabular}

agreement between the computed average and the simulated one as $\varepsilon_{1}<12 \%$. The approximation of the standard deviation is even more accurate since $\varepsilon_{2} \leq 1 \%$. This feature confirms that the standard deviation, unlike the average, is a statistical parameter which is insensitive to errors caused by offsets. The stochastic method thus yields a suitable estimation of the dispersion of the values of the voltage around its average.

The discrepancies between the simulations and the measurements stem most probably from the approximations that are made in the numerical model. First, the port region is modeled here by a $2 \mathrm{~cm}$ gap over the ground plane, whereas in the experiments it corresponds to a port connected to a coaxial cable underneath the PEC plate. Further, the characteristics of the electromagnetic source are still being investigated, in order to choose a more suitable model for the incident field. The transmission-line is not really located in the far-field region of the source antenna. Moreover, a correction factor needs to be assigned to the device which measures the induced voltage, and a loss factor needs to be included to account for the dispersion caused by the PVC tubes. Regarding the stochastic parametrization, we are currently working on developing a more accurate representation of the variations of this geometry. This is done by analyzing the video-recordings of the measurement setup. The application of all these corrections is likely to improve the simulated results, and lead thus to better predictions of the dispersion of the values of the voltage.

\section{CONCLUSIONS}

We have presented a comparison between a stochastic numerical model and an experimental setup. The generic model used in the simulations employs a random Fourier representation of the geometry and a plane-wave model for the incident field. The experimental setup was then described, and several measurements were performed. The statistical postprocessing of these measured results showed that the module of the induced voltage resembled a random variable with a Rician distribution. The preliminary results are encouraging and yield a satisfactory estimation of the average and the variance of the module of the voltage. The accuracy of these results can be improved by taking additional details of the measurement setup into account, and by refining the geometrical description of the wire movement. The efforts that are currently made to improve the accuracy of the numerical model will lead to a better agreement between the simulations and the measurements.

\section{ACKNOWLEDGMENT}

This work is funded by the Dutch Ministry of Economic Affairs, in the Innovation Research Program (IOP) number EMVT 04302.

\section{References}

1 Hill, D.A.(1998), Plane wave integral representation for field in reverberation chambers, IEEE Trans. EMC., 40(3), 209-217.

2 Bellan, D., Pignari,S.(2001), A probabilistic model for the response of an electrically short two-conductor transmission line driven by a random plane wave field, 43(2), 130-139.

3 Sy, O.O., Vaessen, J.A.H.M., Beurden M.C.v., Tijhuis, A.G., Michielsen, B.L.(2007) Probabilistic study of the coupling between deterministic electromagnetic fields and a stochastic thin-wire over a pec plane, paper presented at International Conference on Electromagnetics in Advanced Applications Torino, Italy, 637-640.

4 Krommer, A. R., Ueberhuber, C. W.(1998), Computational Integration, SIAM.

5 Stark H. , Woods J.W.(1986) Probability, random processes, and estimation theory for engineers Prentice-Hall, Englewood Cliffs, New Jersey 